\begin{tabular}{c} 
International Journal of Physical Research, $4(2)(2016) 43-47$ \\
International Journal of Physical Research \\
WPC \\
Website $\begin{array}{c}\text { www.sciencepubco.com/index.php/IJPR } \\
\text { doi: } 10.14419 / \text { ijpr.v4i2.6045 } \\
\text { Research paper }\end{array}$ \\
\hline
\end{tabular}

\title{
Transverse vibration analysis of FGM plates with in-plane exponentially non-homogeneous material
}

\author{
Sayad Boreyri ${ }^{1}$, Mohamad Javad Ketabdari ${ }^{* 1}$, Pouya Mohtat ${ }^{2}$, Ali Moosavi ${ }^{3}$ \\ ${ }^{1}$ Department of Marine Technology, Amirkabir University of Technology, Tehran, Iran \\ ${ }^{2}$ Department of Mechanical Engineering, Sharif University of Technology, Tehran, Iran \\ ${ }^{3}$ Department of Mathematics, Sharif University of Technology, Tehran, Iran \\ *Corresponding author E-mail: ketabdar@aut.ac.ir
}

\begin{abstract}
In this research, free vibration of rectangular functionally graded (FG) plates with in-plane exponentially non-homogeneous material is investigated. Young's modulus and mass density are assumed to vary between a metal-rich and a ceramic-rich zone along one in-plane direction of the plate.

The governing differential equation is derived for the case, and a truncated Taylor series expansion technique is utilized to calculate natural frequencies. A Levy-type solution is obtained for plates having two simply supported edges parallel with the material gradient direction. Results for normalized natural frequency are compared with the 4th order Runge-Kutta method, and when possible with exact solution, showing an accurate agreement. Furthermore, a comprehensive parametric study is carried out to determine the effects of different boundary conditions, aspect ratios, and material variations on the free vibration of FGM plates.
\end{abstract}

\section{Nomenclature}

$c_{1}, c_{2}$ : non-homogeneity parameters of module of elasticity and density, respectively.

$C, F, S$ : Clamped, free, and simply supported edges of the plate, respectively.

$D(\mathrm{x}), E(\mathrm{x}), \rho(\mathrm{x})$ : flexural rigidity, module of elasticity and density of the material.

$D_{0}, E_{0}, \rho_{0}$ : Reference values of $\mathrm{D}(\mathrm{x}), \mathrm{E}(\mathrm{x})$ and $\rho(\mathrm{x})$ at $\mathrm{x}=0$, respectively.

$N$ : number of terms in the truncated Taylor series expansion.

$X(\mathrm{x})$; Displacement (shape) function along $\mathrm{x}$.

$\bar{x}, \bar{y}, \bar{w}$ : Non-dimensional $\mathrm{x}$ and $\mathrm{y}$ direction, and deflection of the plate along $\mathrm{z}$ direction, respectively.

$\omega, \beta$ : Natural frequency and normalized natural frequency, respectively.

$a, b, \lambda, h$ : Length along $\mathrm{x}$ and $\mathrm{y}$ direction, aspect ratio, and thickness of the plate, respectively.

Keywords: FGM Plate; In-Plane Inhomogeneity; Free Vibration; Semi-Analytical Solution.

\section{Introduction}

Composite materials are manufactured based on different industrial needs to optimize the response to external loads and reduce the residual and thermal stresses at desired regions of structures. Functionally graded materials (FGMs) are a class of composites with spatially continuous variation of mechanical properties along one or more directions. This has been achieved by gradually changing the composition of the constituent materials, usually ceramics and metals. Therefore, dealing with interfacial stress concentrations can be avoided. Due to their applicability, FGMs have garnered the attention of many researchers in different structures such as beams [1-3] and plates [4-6].

Vibration analysis of structures with directionally - but not through the thickness - FGM structures is of significance importance. This has been addressed by in [7-9] for axially graded Euler-Bernoulli and Timoshenko beams. It has also been fully discussed for radially FGM circular plates by Sahraee [10], Shariyat and Alipour [11] and Hosseini-Hashemi et al. [12], [13]. How- ever, information about rectangular plates with in-plane inhomogeneity is very limited.

Fundamental frequencies of FGM rectangular plates with in-plane material inhomogeneity was studied by Liu et al. [14] using the Fourier series expansion and an integration technique. Uymaz et al. [15] presented natural frequencies for classical and higher order plates by the Ritz method. Both studies are limited to assuming a power form of the in-plane direction for changes in material properties. Therefore, due to lack of a wide range of data for in-plane inhomogeneous plates, it is needed to thoroughly investigate this problem from other aspects.

In this paper, we investigated vibration characteristics of a rectangular FGM plate with in-plane exponentially non-homogeneous material. Section 2, introduces the theory and methodology of the problem. The equation of motion for the exponentially FGM plate with in-plane exponentially non-homogeneous material is also derived in this section. Results are provided, and the accuracy of the method is investigated in section 3 . Thereafter, a parametric study is performed to analyze the effects of different parameters including boundary conditions, material inhomogeneity and aspect 
ratios on the frequency of the plate. Finally, the authors draw a conclusion based on the most important results.

\section{Theory and methodology}

In this section the theory and boundary conditions of the problem is explained.

\subsection{Finite Taylor series expansion technique}

The present method is a semi-analytical technique based on the truncated Taylor series expansion, with a recursive formulation resulting in reduction of time calculation. Based on the truncated Taylor series expansion, a function $f(x)$ can be approximately replaced by Eq. (1) near a point $\mathrm{x}=\mathrm{x}_{0}$ :

$f(x)=\sum_{k=0}^{N}\left(x-x_{0}\right)^{k} F(k)$

Where $\mathrm{F}(\mathrm{k})$ is referred to the $\mathrm{k}^{\text {th }}$ order differential transform of the function $f(x)$ and is defined as:

$$
F(k)=\frac{1}{k !}\left[\frac{d^{k} f}{d y^{k}}\right]_{x=x_{0}}
$$

Accordingly, the inverse transform of Eq. (2) is expressed as Eq. (1), where uppercase and lowercase letters indicate the transformed function $F(k)$ and the original function $f(x)$, respectively. Equation (1) is approximately true, if the terms $\sum_{k=N+1}^{\infty}(x-$ $\left.x_{0}\right)^{k} F(k)$ become negligible. The appropriate value of $\mathrm{N}$ in Eq. (1) depends on the rate of convergence of the series and the desired accuracy.

Table 1 presents fundamental relations derived directly from the definition of the Taylor series expansion [16,17].

Table 1: Basic Rules for Transforming of Functions by Taylor Series Expansion

\begin{tabular}{|c|c|}
\hline Original Function & Transformed Function \\
\hline$f(x)=\alpha u(x) \pm \beta v(x)$ & $F(k)=\alpha U(k) \pm \beta V(k)$ \\
\hline$f(x)=u(x) v(x)$ & $F(k)=\sum_{n=0}^{k} U(n) V(k-n)$ \\
\hline$f(x)=\frac{d^{m} u(x)}{d x^{m}}$ & $F(k)=(k+1)(k+2) \ldots(k+m) U(k+m)$ \\
\hline$f(x)=x^{m}$ & $\boldsymbol{\delta}(\boldsymbol{k}-\boldsymbol{m})$ \\
\hline$f(x)=\exp (\lambda x)$ & $\frac{\lambda^{k}}{k !}$ \\
\hline
\end{tabular}

\subsection{Plate constitutive equation}

The constitutive equation of motion of a plate can be written as:

$$
\begin{aligned}
& D\left(\nabla^{4} W\right)+2 \frac{\partial D}{\partial x} \frac{\partial}{\partial x}\left(\nabla^{2} W\right)+2 \frac{\partial D}{\partial y} \frac{\partial}{\partial y}\left(\nabla^{2} W\right)+\left(\nabla^{2} D\right)\left(\nabla^{2} W\right)- \\
& (1-v)\left(\frac{\partial^{2} D}{\partial x^{2}} \frac{\partial^{2} W}{\partial y^{2}}-2 \frac{\partial^{2} D}{\partial x \partial y} \frac{\partial^{2} W}{\partial x \partial y}+\frac{\partial^{2} D}{\partial y^{2}} \frac{\partial^{2} W}{\partial x^{2}}\right)+ \\
& \rho h \frac{\partial^{2} W}{\partial t^{2}}=f(x, y, t)
\end{aligned}
$$

In which $D=\frac{E h^{3}}{12\left(1-v^{2}\right)}$ is the flexural rigidity, and $h, E, \rho, v$ are thickness, Young's modulus, density and Poisson's ratio of the material, respectively. For harmonic free vibration of the plate one can set $W(x, y, t)=w(x, y) \sin (\omega t)$, where $\omega$ is the natural frequency. Therefore for free vibration analysis, Eq. (3) can be reduced to:

$$
\begin{aligned}
& D\left(\nabla^{4} w\right)+2 \frac{\partial D}{\partial x} \frac{\partial}{\partial x}\left(\nabla^{2} w\right)+2 \frac{\partial D}{\partial y} \frac{\partial}{\partial y}\left(\nabla^{2} w\right)+\left(\nabla^{2} D\right)\left(\nabla^{2} w\right)- \\
& (1-v)\left(\frac{\partial^{2} D}{\partial x^{2}} \frac{\partial^{2} w}{\partial y^{2}}-2 \frac{\partial^{2} D}{\partial x \partial y} \frac{\partial^{2} w}{\partial x \partial y}+\frac{\partial^{2} D}{\partial y^{2}} \frac{\partial^{2} w}{\partial x^{2}}\right)-\rho h \omega^{2} w=0
\end{aligned}
$$

In this study, a plate with in-plane non-homogeneous material was investigated. Changing along only one direction was taken into account in order to consider limitations of construction. Without loss of generality, we assumed $E$ and $\rho$ change along the $x$ direction (see Fig. 1), and based on exponential functions as:

$$
E=E(x)=E_{0} e^{c_{1} x} \quad \rho=\rho(x)=\rho_{0} e^{c_{2} x} .
$$

Here, $E_{0}$ and $\rho_{0}$ are reference values of $E, \rho$ at $x=0$. A metalrich or ceramic-rich region can be obtained by properly setting values of $E_{0}, \rho_{0}, c_{1}$ and $c_{2}$ in Eq. (5). This method is adopted and fully discussed by Li et al. [18] for beams. Therefore flexural rigidity, $D$, is also a function of $x$, with constant $D_{0}$ as a reference value at $x=0$ as

$$
D=D_{0} e^{c_{1} x} \text { and } D_{0}=\frac{E_{0} h^{3}}{12(1-v)^{2}}
$$

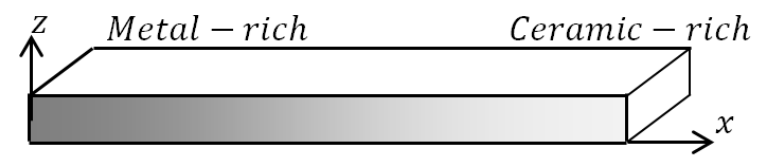

Fig. 1: Changes of Material Properties along One in-Plane Direction.

Variations of $v$ are usually negligible, and it can be considered as constant [19]; hence for an FGM plate with in-plane inhomogeneity, Eq. (4) can be simplified as:

$$
\begin{aligned}
& D(x)\left(\nabla^{4} w\right)+2 c_{1} D(x) \frac{\partial}{\partial x}\left(\nabla^{2} w\right)+c_{1}{ }^{2} D(x)\left(\nabla^{2} w\right)- \\
& (1-v) c_{1}{ }^{2} D(x) \frac{\partial^{2} w}{\partial y^{2}}-\rho(x) h \omega^{2} w=0
\end{aligned}
$$

It should be mentioned that the neutral plane location is different from the mid plane location in plates with material changes along the thickness [20,21], the thing which is not true for plates with inplane changes.

\subsection{Normalization}

Introducing the following non-dimensional parameters

$\bar{x}=x / a \quad \bar{y}=y / b \quad \bar{w}=w / h \quad \lambda=a / b$

Eq. (7) can be rewritten as:

$D_{0} e^{c_{1} a \bar{x}}\left(\frac{h}{a^{4}} \frac{\partial^{4} \bar{w}}{\partial \bar{x}^{4}}+2 \frac{h}{a^{2} b^{2}} \frac{\partial^{4} \bar{w}}{\partial \bar{x}^{2} \partial \bar{y}^{2}}+\frac{h}{b^{4}} \frac{\partial^{4} \bar{w}}{\partial \bar{y}^{4}}\right)+$ $2 D_{0} c_{1} e^{c_{1} a \bar{x}}\left(\frac{h}{a^{3}} \frac{\partial^{3} \bar{w}}{\partial \bar{x}^{3}}+\frac{h}{a b^{2}} \frac{\partial^{3} \bar{w}}{\partial \bar{x} \partial \bar{y}^{2}}\right)+D_{0} c_{1}^{2} e^{c_{1} a \bar{x}}\left(\frac{h}{a^{2}} \frac{\partial^{2} \bar{w}}{\partial \bar{x}^{2}}+\right.$ $\left.\frac{h}{b^{2}} \frac{\partial^{2} \bar{w}}{\partial \bar{y}^{2}}\right)-(1-v) D_{0} c_{1}^{2} e^{c_{1} a \bar{x}} \frac{h}{b^{2}} \frac{\partial^{2} \bar{w}}{\partial \bar{y}^{2}}-\rho_{0} e^{c_{2} a \bar{x}} h^{2} \omega^{2} \bar{w}=0$

Where $\lambda, a$ and $b$ are aspect ratio, length and width of the plate, respectively. The governing equation for free vibration of FG plates with in-plane material inhomogeneity can be simplified to a non-dimensional form as:

$\left(\frac{\partial^{4} \bar{w}}{\partial \bar{x}^{4}}+2 \lambda^{2} \frac{\partial^{4} \bar{w}}{\partial \bar{x}^{2} \partial \bar{y}^{2}}+\lambda^{4} \frac{\partial^{4} \bar{w}}{\partial \bar{y}^{4}}\right)+2 c_{1} a\left(\frac{\partial^{3} \bar{w}}{\partial \bar{x}^{3}}+\lambda^{2} \frac{\partial^{3} \bar{w}}{\partial \bar{x} \partial \bar{y}^{2}}\right)+$ $a^{2}\left(c_{1}^{2} \frac{\partial^{2} \bar{w}}{\partial \bar{x}^{2}}+v \lambda^{2} \frac{\partial^{2} \bar{w}}{\partial \bar{y}^{2}}\right)-\beta^{2} \bar{w}=0$

Where

$\beta^{2}=\frac{12(1-v)^{2} a^{4} \rho_{0} \omega^{2}}{E_{0} h^{2}}$.

Considering two opposite edges, $\bar{y}=0$ and $\bar{y}=1$, simply supported (Levy-type solution), one can write

$\bar{w}(\bar{x}, \bar{y})=X(\bar{x}) \sin (m \pi \bar{y})$.

In the remaining of the paper the bar $\left(^{-}\right)$symbol will not be shown for the sake of simplicity. Equation (10) can then be simplified as: 
$X^{(4)}+A_{1} X^{\prime \prime \prime}+A_{2} X^{\prime \prime}+A_{3} X^{\prime}+\left[A_{4}-\beta^{2} e^{-a x\left(c_{1}-c_{2}\right)}\right] X=0(11)$

Where

$A_{1}=2 c_{1} a \quad, A_{2}=a^{2} c_{1}^{2}-2(m \pi \lambda)^{2}$

$A_{3}=-2 c_{1} a(m \pi \lambda)^{2}, A_{4}=(m \pi \lambda)^{4}-v\left(c_{1} a m \pi \lambda\right)^{2}$

Applying the differential transform method presented in subsection 2.1 Oto Eq. (11) gives the following relation.

$\left(\prod_{i=1}^{4}(k+i)\right) X(k+4)+A_{1} \cdot\left(\prod_{i=1}^{3}(k+i)\right) X(k+3)+$ $A_{2} \cdot\left(\prod_{i=1}^{2}(k+i)\right) X(k+2)+A_{3} \cdot(k+1) X(k+1)+A_{4} X(k)-$ $\beta^{2} \sum_{n=0}^{k} \frac{\left[a\left(c_{2}-c_{1}\right)\right]^{n}}{n !} X(k-n)=0$

Therefore, a recursive formula can be obtained by rearranging the above equation.

$$
\begin{aligned}
& X(k+4)=-\frac{A_{1}}{(k+4)} X(k+3)-\frac{A_{2}}{(k+4)(k+3)} X(k+2)- \\
& \frac{A_{3}}{(k+4)(k+3)(k+2)} X(k+1)-\frac{A_{4}}{\prod_{i=1}^{4}(k+i)} X(k)+ \\
& \frac{\beta^{2}}{\prod_{i=1}^{4}(k+i)} \sum_{n=0}^{k} \frac{\left[a\left(c_{2}-c_{1}\right)\right]^{n}}{n !} X(k-n)
\end{aligned}
$$

Solving the problem depends on the recursive formula of Eq. (13) for $k=0$ or (4). Two of the four terms $X(0), X(1), X(2)$ and $X(3)$ are given by boundary conditions, and the two remaining terms form a matrix. Setting the determinant of the coefficients to zero gives the natural frequency, $\beta$.

\section{Results and discussion}

In this section, validation of the model and results are presented.

\subsection{Validation}

For the situation where non-homogeneity parameters $\left(c_{1}, c_{2}\right)$ in Eq. (13) are set to zero, analytical solutions are available. In Table 2 , a comparison was made between the DTM, $4^{\text {th }}$ order RungeKutta and those from analytical solution by Leissa [22].

It can be seen from Table 2 that the present method is precise in calculating frequencies, while RK4 demonstrates some small variations. Moreover, it is worth mentioning that obtaining more accurate results from the RK4 took a few days, while DTM results

\begin{tabular}{|c|c|c|c|c|}
\hline B.Cs. & method & $\begin{array}{l}\text { Mode } \\
1 \text { st }\end{array}$ & 2nd & $3 \mathrm{rd}$ \\
\hline \multirow{3}{*}{ SCSC } & Exact & 28.9509 & 54.7431 & 69.3270 \\
\hline & DTM & 28.9509 & 54.7430 & 69.3270 \\
\hline & RK4 & 28.9695 & 54.7531 & 69.7485 \\
\hline \multirow{3}{*}{ SCSS } & Exact & 23.6463 & 51.6743 & 58.6464 \\
\hline & DTM & 23.6463 & 51.6743 & 58.6463 \\
\hline & RK4 & 23.6530 & 51.6790 & 58.8883 \\
\hline \multirow{3}{*}{ SSSS } & Exact & 19.7392 & 49.3480 & 49.3480 \\
\hline & DTM & 19.7392 & 49.3480 & 49.3480 \\
\hline & RK4 & 19.7415 & 49.3503 & 49.4791 \\
\hline \multirow{3}{*}{ SCSF } & Exact & 12.6874 & 33.0651 & 41.7019 \\
\hline & DTM & 12.6874 & 33.0651 & 41.7019 \\
\hline & RK4 & 12.6875 & 33.0926 & 41.7220 \\
\hline \multirow{3}{*}{ SFSS } & Exact & 11.6845 & 27.7563 & 41.1967 \\
\hline & DTM & 11.6845 & 27.7563 & 41.1966 \\
\hline & RK4 & 11.6845 & 27.7687 & 41.1966 \\
\hline \multirow{3}{*}{ SFSF } & Exact & 9.6314 & 16.1348 & 36.7256 \\
\hline & DTM & 9.6313 & 16.1348 & 36.7256 \\
\hline & RK4 & 9.6313 & 16.1351 & 36.7648 \\
\hline
\end{tabular}
were easily achievable in a few hours on the same computer.
Table 2: Natural Frequencies of Homogeneous Square Plates

The present method is semi-analytical, and results are dependent on the number of terms, $\mathrm{N}$, in finite Taylor series expansion of Eq.(1). Here, Fig. 2 and Fig. 3 are presented to check the convergence of solutions. They consider three different values for $c_{1}$ and $c_{2}$ for FG plates with SCSC and SFSF boundary conditions, respectively. Convergence diagrams for other boundary conditions are the same and have not been presented.

In addition, They show that for larger values of non-homogeneity parameters, $c_{1}$ and $c_{2}$, more terms should be considered to obtain desired accuracy. For instance if the first natural frequency is considered, $N=20$ is a proper value for $\left(c_{1}, c_{2}\right)=(0,0)$--Fig. 2-a and Fig. 3-a -- but it is not sufficient when $\left(c_{1}, c_{2}\right)=(1,0.5)$, see Fig. 2-b and Fig. 3-b. Moreover as it is expected, obtaining higher natural frequencies needs consideration of higher values of $N$.

\subsection{Parametric studies}

In Table 3 to Table 8, the first three natural frequencies of FGM plates are presented for SSSS, SCSC, SFSF, SCSS, SFSS, and SCSF boundary conditions for three different aspect ratios and with non-zero values of $\boldsymbol{c}_{\mathbf{1}}$ and $\boldsymbol{c}_{\mathbf{2}}$. Changes in natural frequency versus aspect ratio, in-plane non-homogeneous parameters and different boundary conditions are presented for the first three vibrational modes of the plate in these tables.
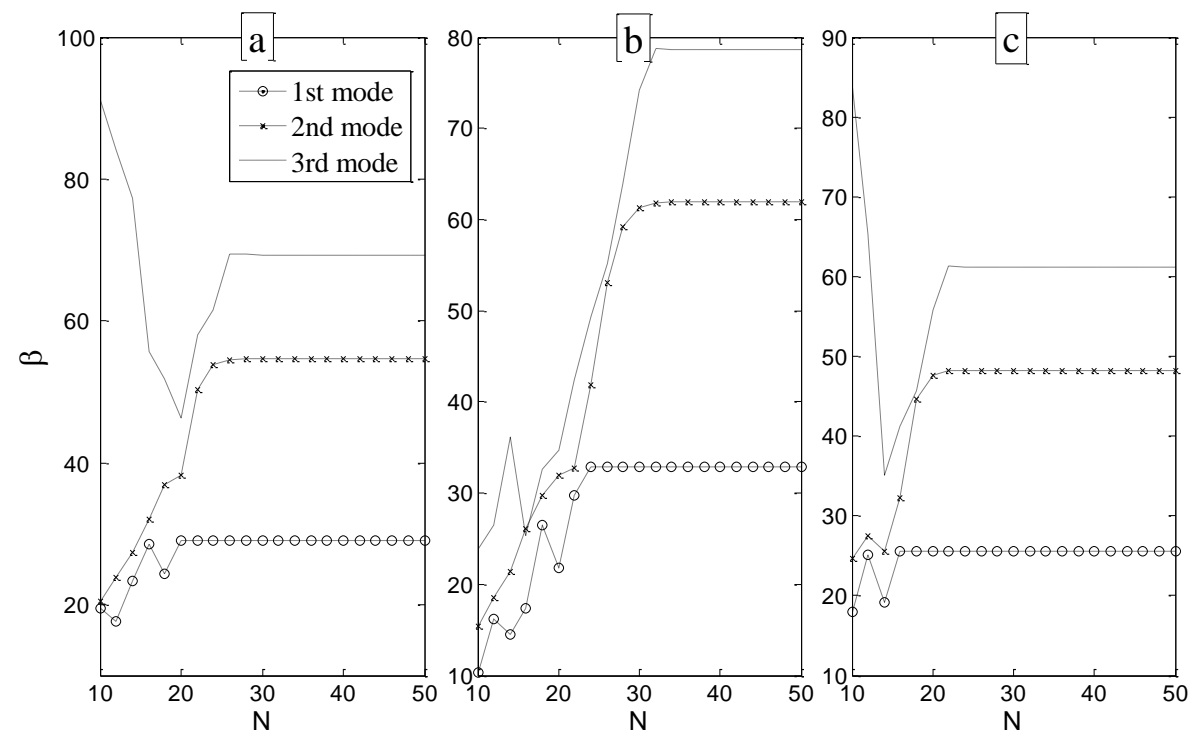

Fig. 2: Convergence of the First Three Natural Frequencies vs. N, Number of Terms, for FGM SCSC Plate for $\left(c_{1}, c_{2}\right)=\mathrm{A}:(0,0)$, B: $(1,0.5)$, C: $(-1,-0.5)$. 


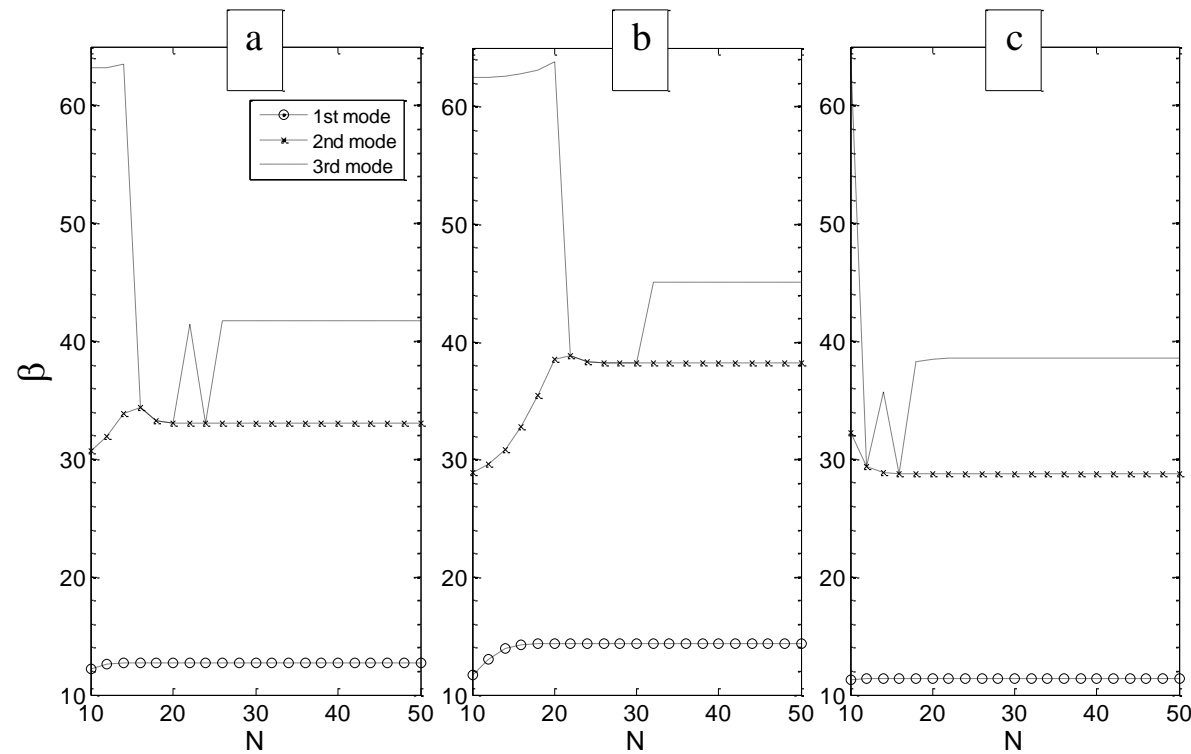

Fig. 3: Convergence of the First Three Natural Frequencies vs. N, Number of Terms, for FGM SFSF Plate for $\left(c_{1}, c_{2}\right)=\mathrm{A}:(0,0), \mathrm{B}:(1,0.5)$, C: $(-1,-0.5)$.

Table 3: 1st Three Frequency Parameters of the FGM SSSS Plate

\begin{tabular}{|c|c|c|c|c|c|c|c|c|c|}
\hline & $\lambda=1$ & & & $\lambda=1.5$ & & & $\lambda=2$ & & \\
\hline$\left(c_{1}, c_{2}\right)$ & $(0,0)$ & $(1,0.5)$ & $(-1,-0.5)$ & $(0,0)$ & $(1,0.5)$ & $(-1,-0.5)$ & $(0,0)$ & $(1,0.5)$ & $(-1,-0.5)$ \\
\hline$\omega_{1}$ & 19.739 & 22.425 & 17.465 & 32.076 & 36.399 & 28.347 & 49.348 & 55.870 & 43.512 \\
\hline$\omega_{2}$ & 49.348 & 55.947 & 43.572 & 61.685 & 69.959 & 54.484 & 78.957 & 89.571 & 69.758 \\
\hline
\end{tabular}

Table 4: 1 st Three Frequency Parameters of the FGM SCSC Plate

\begin{tabular}{|c|c|c|c|c|c|c|c|c|c|}
\hline & $\lambda=1$ & & & $\lambda=1.5$ & & & $\lambda=2$ & & \\
\hline$\left(c_{1}, c_{2}\right)$ & $(0,0)$ & $(1,0.5)$ & $(-1,-0.5)$ & $(0,0)$ & $(1,0.5)$ & $(-1,-0.5)$ & $(0,0)$ & $(1,0.5)$ & $(-1,-0.5)$ \\
\hline$\omega_{1}$ & 28.951 & 32.897 & 25.620 & 39.089 & 44.339 & 34.531 & 54.743 & 61.987 & 48.276 \\
\hline$\omega_{2}$ & 54.743 & 61.987 & 48.276 & 79.525 & 90.176 & 70.229 & 94.585 & 107.223 & 83.505 \\
\hline$\omega_{3}$ & 69.327 & 78.641 & 61.246 & 102.216 & 115.358 & 89.841 & 154.776 & 175.370 & 136.578 \\
\hline
\end{tabular}

Table 5: 1st Three Frequency Parameters of the FGM SFSF Plate

\begin{tabular}{|c|c|c|c|c|c|c|c|c|c|}
\hline & $\lambda=1$ & & & $\lambda=1.5$ & & & $\lambda=2$ & & \\
\hline$\left(c_{1}, c_{2}\right)$ & $(0,0)$ & $(1,0.5)$ & $(-1,-0.5)$ & $(0,0)$ & $(1,0.5)$ & $(-1,-0.5)$ & $(0,0)$ & $(1,0.5)$ & $(-1,-0.5)$ \\
\hline$\omega_{1}$ & 9.631 & 10.980 & 8.551 & 21.095 & 23.932 & 18.638 & 37.958 & 42.010 & 32.718 \\
\hline$\omega_{2}$ & 16.135 & 18.419 & 14.344 & 25.311 & 28.800 & 22.429 & 40.866 & 47.702 & 37.151 \\
\hline
\end{tabular}

Table 6: 1 st Three Frequency Parameters of the FGM SCSS Plate

\begin{tabular}{|c|c|c|c|c|c|c|c|c|c|}
\hline & $\lambda=1$ & & & $\lambda=1.5$ & & & $\lambda=2$ & & \\
\hline$\left(c_{1}, c_{2}\right)$ & $(0,0)$ & $(1,0.5)$ & $(-1,-0.5)$ & $(0,0)$ & $(1,0.5)$ & $(-1,-0.5)$ & $(0,0)$ & $(1,0.5)$ & $(-1,-0.5)$ \\
\hline$\omega_{1}$ & 23.646 & 26.543 & 21.175 & 35.051 & 39.822 & 30.929 & 51.674 & 58.865 & 45.298 \\
\hline$\omega_{2}$ & 51.674 & 58.865 & 45.298 & 69.913 & 78.986 & 61.973 & 86.134 & 97.669 & 76.069 \\
\hline$\omega_{3}$ & 58.646 & 65.939 & 52.241 & 100.270 & 113.874 & 87.455 & 140.846 & 159.367 & 124.473 \\
\hline
\end{tabular}

Table 7: 1st Three Frequency Parameters of the FGM SFSS Plate

\begin{tabular}{|c|c|c|c|c|c|c|c|c|c|}
\hline & $\lambda=1$ & & & $\lambda=1.5$ & & & $\lambda=2$ & & \\
\hline$\left(c_{1}, c_{2}\right)$ & $(0,0)$ & $(1,0.5)$ & $(-1,-0.5)$ & $(0,0)$ & $(1,0.5)$ & $(-1,-0.5)$ & $(0,0)$ & $(1,0.5)$ & $(-1,-0.5)$ \\
\hline$\omega_{1}$ & 11.685 & 13.121 & 10.539 & 22.582 & 24.935 & 20.592 & 39.075 & 42.145 & 36.495 \\
\hline$\omega_{2}$ & 27.756 & 31.735 & 24.415 & 38.252 & 44.171 & 33.096 & 54.195 & 62.551 & 46.741 \\
\hline$\omega_{3}$ & 41.197 & 44.670 & 37.889 & 72.718 & 83.234 & 63.490 & 88.553 & 101.554 & 77.102 \\
\hline
\end{tabular}

Table 8: 1 st Three Frequency Parameters of the FGM SCSF Plate

\begin{tabular}{|c|c|c|c|c|c|c|c|c|c|}
\hline & $\lambda=1$ & & & $\lambda=1.5$ & & & $\lambda=2$ & & \\
\hline$\left(c_{1}, c_{2}\right)$ & $(0,0)$ & $(1,0.5)$ & $(-1,-0.5)$ & $(0,0)$ & $(1,0.5)$ & $(-1,-0.5)$ & $(0,0)$ & $(1,0.5)$ & $(-1,-0.5)$ \\
\hline$\omega_{1}$ & 12.687 & 14.569 & 11.199 & 22.958 & 26.829 & 19.771 & 39.246 & 47.069 & 32.955 \\
\hline$\omega_{2}$ & 33.065 & 36.921 & 29.777 & 42.516 & 47.185 & 38.265 & 57.508 & 63.887 & 51.507 \\
\hline
\end{tabular}

\section{Conclusion}

In this research, the formulation for plates with exponential gradient of the material and having levy-type boundary conditions was derived. A semi-analytical solution, truncated Taylor series expansion technique, was employed to obtain approximate but highly accurate solution for free vibration of the FGM rectangular plate. A number of comparisons were made with the fourth order Runge-
Kutta method and those available in the literature, showing an excellent agreement. Finally, the dependency of the free vibration of plates on in-plane FG properties, boundary conditions and aspect ratios were investigated. All semi-analytical results presented in this paper may serve to validate other analytical and numerical methods. In addition and in the light of results the following conclusions can be drawn: 
- Natural frequency is directly related to the value of nonhomogeneous parameters, $c_{1}$ and $c_{2}$, and increases with their increment;

- Larger number of terms, N, in the finite Taylor series expansion should be taken for higher values of nonhomogeneous parameters, $c_{1}$ and $c_{2}$, which is also directly proportioned to the desired vibrational modes;

- Natural frequency has a direct relation with aspect ratio for all in-plane non-homogeneous parameters.

\section{References}

[1] K. Suddoung, J. Charoensuk, N. Wattanasakulpong, Vibration response of stepped FGM beams with elastically end constraints using differential transformation method, Applied Acoustics 77 (2014) 20-28. http://dx.doi.org/10.1016/j.apacoust.2013.09.018.

[2] A.M. Zenkour, I.A. Abbas, Free vibration analysis of doubly convex/concave functionally graded sandwich beams, Journal of Vibroengineering 16 (2014) 2747-2755.

[3] V. Azadi, M. Azadi, S.A. Fazelzadeh, E. Azadi, Active control of an fgm beam under follower force with piezoelectric sensors/actuators, International Journal of Structural Stability and Dynamics $\quad 14 \quad$ (2014) 1-19. http://dx.doi.org/10.1142/S0219455413500636.

[4] T.Y. Ng, K.Y. Lam, K.M. Liew, Effects of FGM materials on the parametric resonance of plate structures, Comput. Methods Appl. Mech. $\quad$ Engrg $\quad 190 \quad$ (2000) 953-962. http://dx.doi.org/10.1016/S0045-7825(99)00455-7.

[5] M.J. Ketabdari, A. Allahverdi, S. Boreyri, M. Fadavi Ardestani, Free vibration analysis of homogeneous and FGM Skew plates resting on variable Winkler-Pasternak elastic foundation, Mechanics \& Industry (2015) 1-11.

[6] Y.S. Hamed, Nonlinear oscillations and chaotic dynamics of a sup ported FGM rectangular plate system under mixed excitations, Journal of Vibroengineering 16 (2014) 3218-3235.

[7] a. Shahba, R. Attarnejad, M.T. Marvi, S. Hajilar, Free vibration and stability analysis of axially functionally graded tapered Timoshenko beams with classical and non-classical boundary conditions, Composites Part B: Engineering 42 (2011) 801-808. http://dx.doi.org/10.1016/j.compositesb.2011.01.017.

[8] H. Hein, L. Feklistova, Free vibrations of non-uniform and axially functionally graded beams using Haar wavelets, Engineering Struc$\begin{array}{lll}\text { tures } & 33 & \text { (2011) 3696-3701. }\end{array}$ http://dx.doi.org/10.1016/j.engstruct.2011.08.006

[9] S. Rajasekaran, Differential transformation and differential quadrature methods for centrifugally stiffened axially functionally graded tapered beams, International Journal of Mechanical Sciences 74 (2013) 15-31. http://dx.doi.org/10.1016/j.ijmecsci.2013.04.004.

[10] S. Sahraee, Bending analysis of functionally graded sectorial plates using Levinson plate theory, Composite Structures 88 (2009) 548557. http://dx.doi.org/10.1016/j.compstruct.2008.05.014.

[11] M. Shariyat, M.M. Alipour, A power series solution for vibration and complex modal stress analyses of variable thickness viscoelastic two-directional FGM circular plates on elastic foundations, Applied Mathematical Modelling 37 (2013) 3063-3076. http://dx.doi.org/10.1016/j.apm.2012.07.037.

[12] S. Hosseini-Hashemi, H. Rokni Damavandi Taher, H. Akhavan, Vibration analysis of radially FGM sectorial plates of variable thickness on elastic foundations, Composite Structures 92 (2010) 1734-1743. http://dx.doi.org/10.1016/j.compstruct.2009.12.016

[13] S. Hosseini-hashemi, H. Akhavan, H.R. Damavandi, N. Daemi, A Alibeigloo, Differential quadrature analysis of functionally graded circular and annular sector plates on elastic foundation, Materials and Design $31 \quad$ (2010) 1871-1880. http://dx.doi.org/10.1016/j.matdes.2009.10.060.

[14] D.Y. Liu, C.Y. Wang, W.Q. Chen, Free vibration of FGM plates with in-plane material inhomogeneity, Composite Structures 92 (2010)

1047-1051. http://dx.doi.org/10.1016/j.compstruct.2009.10.001

[15] B. Uymaz, M. Aydogdu, S. Filiz, Vibration analyses of FGM plates with in-plane material inhomogeneity by Ritz method, Composite $\begin{array}{llll}\text { Structures } & 94 & \text { (2012) }\end{array}$ http://dx.doi.org/10.1016/j.compstruct.2011.11.002.

[16] F. Ayaz, on the two-dimensional differential transform method, Applied Mathematics and Computation 143 (2003) 361-374. http://dx.doi.org/10.1016/S0096-3003(02)00368-5.

[17] S. Boreyri, P. Mohtat, M.J. Ketabdari, A. Moosavi, Vibration analysis of a tapered beam with exponentially varying thickness resting on Winkler foundation using the differential transform method, International Journal of Physical Research 2 (2014) 10-15 http://dx.doi.org/10.14419/ijpr.v2i1.2152.

[18] X.-F. Li, Y.-A. Kang, J.-X. Wu, Exact frequency equations of free vibration of exponentially functionally graded beams, Applied
Acoustics
(2013)
$413-420$ http://dx.doi.org/10.1016/j.apacoust.2012.08.003.

[19] M. Shariyat, M. Alipour, Differential transform vibration and modal stress analyses of circular plates made of two-directional functionally graded materials resting on elastic foundations, Arch Appl Mech 81 (2011) 1289-1306. http://dx.doi.org/10.1007/s00419-0100484-x.

[20] A. Fekrar, M.S.A. Houari, A. Tounsi, S.R. Mahmoud, A new fiveunknown refined theory based on neutral surface position for bending analysis of exponential graded plates, Meccanica 49 (2014) 795-810. http://dx.doi.org/10.1007/s11012-013-9827-3.

[21] A.A. Bousahla, M.S.A. Houari, A. Tounsi, E.A. Adda Bedia, a Novel Higher Order Shear and Normal Deformation Theory Based on Neutral Surface Position for Bending Analysis of Advanced Composite Plates, International Journal of Computational Methods 11 (2014) 1350082. http://dx.doi.org/10.1142/S0219876213500825.

[22] A.W. Leissa, The free vibration of rectangular plates, Journal ofSound and Vibration 31 (1973) 257-293. http://dx.doi.org/10.1016/S0022-460X(73)80371-2. 